\title{
Clinical Usefulness of the Pocket Echocardiographic Device: Potential Advantage and Reliability Compared to the Conventional Echocardiographic Equipment
}

\section{Jiang M, Shen X*, Qiao Z, Zhao H, Wang J and Pu J \\ Department of Cardiology, Shanghai Jiaotong University, China}

*Corresponding author: Xuedong Shen, Department of Cardiology, Renji Hospital, Medical College of Shanghai Jiaotong University, Shanghai 200127, China, Tel: 086-216838-3217; Email: shenxd@hotmail.com

\section{Research Article \\ Volume 2 Issue 2}

Received Date: March 19, 2018

Published Date: April 09, 2018

\section{Abstract}

We hypothesized that pocket echocardiography (PE) is a useful and has a potential advantage in clinical practice.

Methods: Study population consisted of 200 patients who underwent both PE and conventional (CE) (male 107, age $56.6 \pm 16.6$ years). Each patient was submitted to physical examination before echocardiographic assessment. The agreement, sensitivity, specificity between PE and CE were assessed based on the diagnostic results of CE.

Results: About 77.5\% clinical questions could be answered by PE, however, only 6\% questions could be answer by physical examination. The sensitivities and specificities of PE to detect the enlargement of left ventricle, right ventricle, left atrium and right atrium detected by CE were 89\%- 100\% and 100\% (AUC= 0.94- 1.00), respectively. The sensitivities and specificities of PE to detect significant valvular regurgitation by CE were $96 \%$ and $97 \%$ (AUC=0.97), respectively. There was excellent agreement between PE and CE in evaluating severity of valvular regurgitation for the four valves (kappa $=0.905$ ). Eight of 9 patients with congenital heart disease could be successfully diagnosed by PE and confirmed by CE. Eight patients with critical ills in emergency room had been correctly diagnosed by PE, which should be acute managements after diagnosis.

Conclusions: With pocket size nature, good agreement between PE and CE. PE showed additive clinical value over the clinical physical examination. It may alter the current consultation mode in clinical practice in the future.

Keywords: Echocardiography; Pocket echocardiography 


\section{Open Access Journal of Cardiology}

Abbreviations: PE: Pocket Size Echocardiography; CE: Conventional Echocardiography; ROC: Receiver Operating Characteristic; ASD: Atrial Septal Defect.

\section{Introduction}

Echocardiographic examination becomes a crucial diagnostic process in patients with cardiovascular disease. Technological evolution has led to the development of pocket size echocardiography (PE) with basic functions. It had great advantages compared to the full-size comprehensive and conventional echocardiography (CE) [1-4]. PE can be wildly used in bedside examination during ward rounds, in emergency room for cardiology consultation and in remote village for its portable property because of pocket size nature. Since PE is often used by cardiologists but not sonographer to get the first information, it is necessary to assess the clinical value and to assess its contribution in current consultant mode. We hypothesized that PE is a useful and has a potential advantage in clinical practice compared to $\mathrm{CE}$.

\section{Methods}

The study protocol was reviewed and approved by the Ethics Committee of Renji Hospital, Shanghai. We studied 200 patients who underwent PE and CE (male 107, female 93, age 56.6 \pm 16.6 years) during July 1, 2012 to August 31, 2012 in our hospital, included outpatients 105 (52.5\%), emergency patients $33(16.5 \%)$, hospitalized patients 57 (28.5\%) and outside hospital consultation 5 (2.5\%). A GE V-Scan (weight $390 \mathrm{~g}$, General Electric, Milwaukee, WI, USA). Figure 1 with fixed sector angle of $75^{\circ}$ and a 1.7 $3.8 \mathrm{MHz}$ transducer was used for PE. Color flow Imaging is available with a $30 \cong$ maneuverable sector. Spectral Doppler and M-mode imaging is not available. CE was performed with GE vivid E9 and PHILIPS ie33 within 2 hours after PE. Each patient was submitted to physical examination before PE and CE assessment. The patient demographics and underlining disease are listed in the Table 1. Beside 33 patients underwent bedside PE, 26 patients in catheter laboratory and intensive care unit also underwent bedside PE. PE studies were performed by a level 2 cardiologist followed by the guideline issued by American Society of Echocardiography [5]. Images and movies were acquired and stored in a folder of the device. CE was performed by a well trained level 3 sonographer. Each examiner was blinded to the results of the other examination. The final echocardiographic report was based on the results of CE. A single echocardiogrpher reviewed the examinations of all subjects separately to analyze the results.

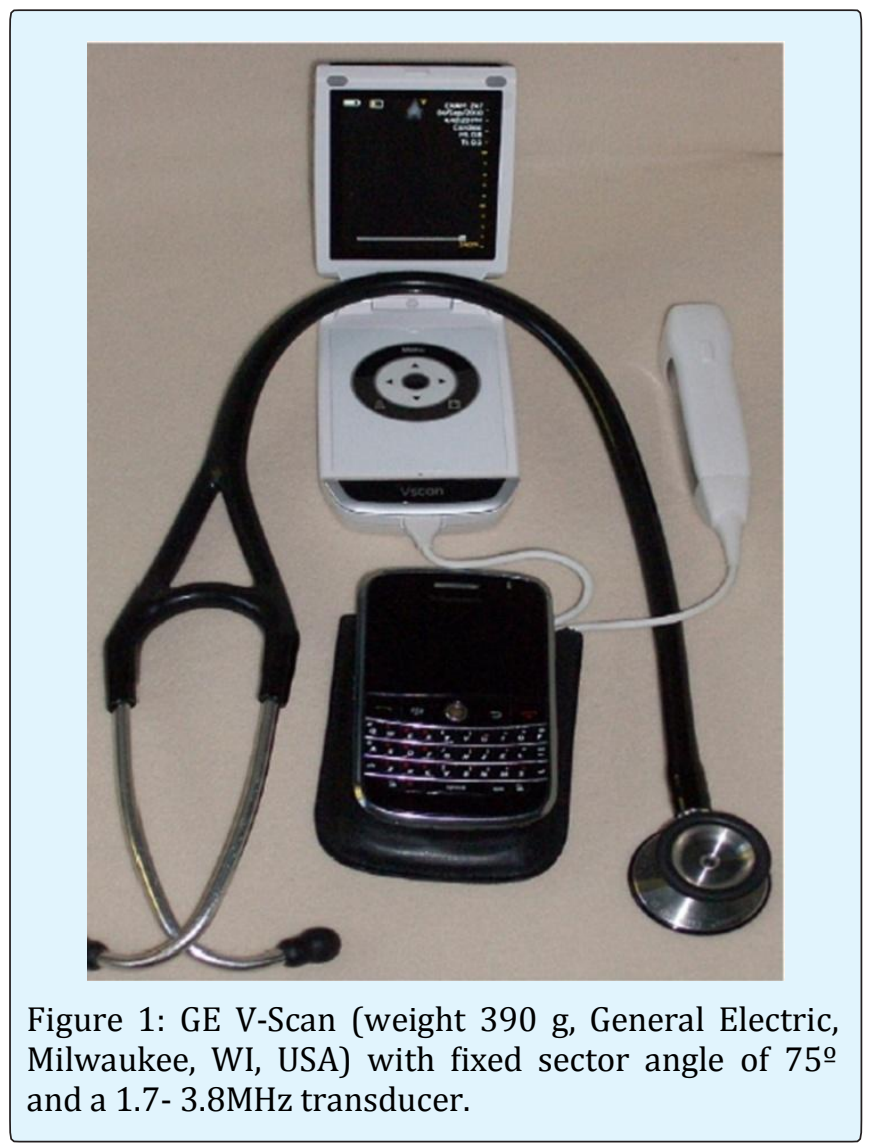

\begin{tabular}{|c|c|}
\hline & Patients \\
\hline Male/Female & $107 / 93$ \\
\hline Age (years) & $56.6 \pm 16.6$ \\
\hline BMI & $23.1 \pm 4.1$ \\
\hline \multicolumn{2}{|l|}{ Underlining diseases } \\
\hline Documented coronary artery disease & $71(35.5 \%)$ \\
\hline Hypertension & $86(43 \%)$ \\
\hline Diabetes mellitus & $30(15 \%)$ \\
\hline Previous valve repairment & $9(4.5 \%)$ \\
\hline Previous prosthetic valve replacement & $10(5 \%)$ \\
\hline $\begin{array}{l}\text { Previous operation for congenital heart } \\
\text { disease }\end{array}$ & $9(4.5 \%)$ \\
\hline Normal subjects & $46(23 \%)$ \\
\hline
\end{tabular}

Table 1: Patient demographics and underlining disease.

Cardiovascular diseases were classified as major and minor abnormalities. Major abnormalities were considered to be clinically significant abnormalities that would trigger further diagnostic evaluation, to change or initiate treatment management, or to have prognostic significance [6]. Minor abnormalities were considered to 


\section{Open Access Journal of Cardiology}

be abnormalities that were of no clinical significance and therefore would not affect decision making for patient management. The present and severity of the valve regurgitation diagnosed by color flow imaging was evaluated by PE according to the guideline issued by American Society of Echocardiography [7]. Unsuspected major abnormalities were considered to be the first detected major abnormalities by echocardiography that did not reported in the history or were not suspected or previously described from the symptoms or physical examination. Since the PE had no spectral Doppler modality, left ventricular diastolic function and pulmonary systolic pressure could not be set to be compared.

Sample size was calculated by SPSS 17.0 using noninferiority tests for two correlated proportions. Continuous variables were presented as mean \pm standard deviation. Categorical data was assessed with a Chisquare or Fisher-exact test if cell sizes were $<5$. Agreement between PE and CE in echocardiographic results was assessed by the Kappa statistics. Based on Landis \& Koch 's classification [8], kappa values 0.21-0.40 looks as "Fair"; 0.41-0.60 looks as "Moderate"; 0.61-0.80 looks as "Substantial" and 0.81-1.00 looks as "Almost perfect". A receiver operating characteristic (ROC) curve was used for evaluation of cutoff value, sensitivity and specificity of PE to predict cardiac chamber dimensions measured by CE. A two-tailed $p$ value $<0.05$ was considered significant.

\section{Results}

\section{Image Quality}

PE and CE were successfully performed on all patients. The percentage of patients with good, regular and bad image quality by PE were $27 \%$ (54/200), 63\% (125/200) and $10 \%(21 / 200)$, respectively. The percentage of patients with good, regular and bad image quality using CE were $26 \%, 69 \%$ and $5 \%$, respectively. There were no significant difference in the image quality between PE and CE $(\mathrm{p}=0.33)$.

\section{The Capability to Answer the Clinical Questions}

PE was able to answer 155/200 (77.5\%) clinical questions (Table 2). Some of the questions could not be answered by PE because of spectral Doppler unavailable $(24 / 200)$ (such as pulmonary hypertension and left ventricular diastolic dysfunction), and bad image quality $(21 / 200)$. However, PE was able to answer 57 of 59 (96.6\%) questions in the cardiac catheter laboratory, intensive care unit and emergency room, included complication after interventional procedure (such as: atrial fibrillation ablation, percutanous coronary intervention and transcatheter inter-atrial or ventricular septal closures), global and segmental cardiac function, significant valvular heart disease, aortic dissection, lower blood volume and pleural effusion (Table 2). The mean examine time using PE was significant shorter (5 min) compared to CE $(45 \mathrm{~min})(\mathrm{p}<0.05)$ and the patient felt comfortable in all PE patients.

\begin{tabular}{|c|c|c|}
\hline Questions & & PE \\
\hline \multirow{2}{*}{$\begin{array}{l}\text { Did the PE answer the questions in } \\
\text { routine clinical practice? }\end{array}$} & Yes & 155 \\
\hline & No & 45 \\
\hline \multirow{2}{*}{$\begin{array}{l}\text { Did the PE answer the clinical question in } \\
\text { the catheter Lab, intensive care unit and } \\
\text { emergency room? }\end{array}$} & Yes & 57 \\
\hline & No & 2 \\
\hline \multirow{2}{*}{ 1. Complication after interventional? } & Yes & 18 \\
\hline & No & 0 \\
\hline \multirow{2}{*}{ 2. Global and segmental cardiac function? } & Yes & 22 \\
\hline & No & 0 \\
\hline \multirow{2}{*}{ 3. Significant valvular heart disease? } & Yes & 5 \\
\hline & No & 0 \\
\hline \multirow{2}{*}{ 4. Aortic dissection? } & Yes & 6 \\
\hline & No & 0 \\
\hline \multirow{2}{*}{ 5. Lower blood volume? } & Yes & 5 \\
\hline & No & 0 \\
\hline \multirow{2}{*}{ 6. Pleural effusion? } & Yes & 1 \\
\hline & No & 0 \\
\hline \multirow{2}{*}{ 7. Pulmonary embolism? } & Yes & 0 \\
\hline & No & 2 \\
\hline
\end{tabular}

Table 2: The capability of PE and CE to answer the clinical questions.

\section{Agreement between PE and CE Results}

The agreement between PE and CE for detection of the major and minor abnormalities lists in Table 3 . As the Table presented that 403 of 419 (96\%) unsuspected major abnormalities detected by CE could be also detected by PE (kappa= 0.962). Only 26 of $419(6 \%)$ major findings could be found by physical examination. There were 377 (90\%) new major abnormalities found by PE that missed by physical examination. There were great agreements for evaluation of cardiac chamber dimensions between PE and CE (kappa $=0.936-1.000)$. The sensitivities and specificities of PE to detect the enlargement of left ventricle, right ventricle, left atrium and right atrium detected by CE were $89 \%-100 \%$ and $100 \%(A U C=0.94-1.00)$, respectively (Table 4). All of 8 


\section{Open Access Journal of Cardiology}

patients with hypertrophic cardiomyopathy could be diagnosed by PE, included 1 patient with apical hypertrophic cardiomyopathy (kappa $=1.000$ ) (Figure 2). The value of PE for evaluation of valvular heart disease was high. There was great agreement on valve stenosis, significant valve regurgitation ( $>$ mild), valvular vegetation (Figure 3) and mitral prolapse (Figure 4) between PE and CE (kappa=0.989-1.000). The severity of valve regurgitation evaluated by $\mathrm{PE}$ was highly agreed with CE Kappa $=0.905$, (Table 5). Ten patients with prosthetic mitral valve replacement could be evaluated by $\mathrm{PE}$, included 1 patient with mitral paravalvular leaking and confirmed by transesophageal echocardiography (Figure 5).
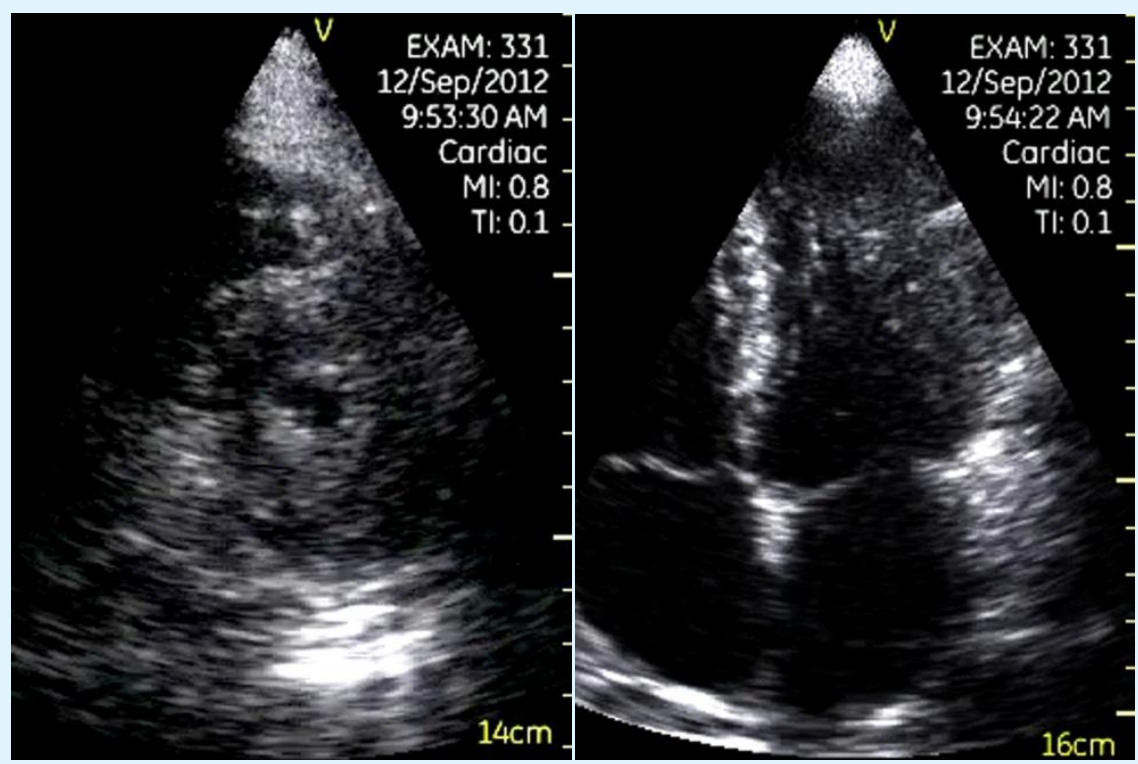

Figure 2: Apical hypertrophic cardiomyopathy. Left: Parasternal short axis view at apical level showed a significant left ventricular hypertrophy in the apical level; Right: Apical four chamber view.

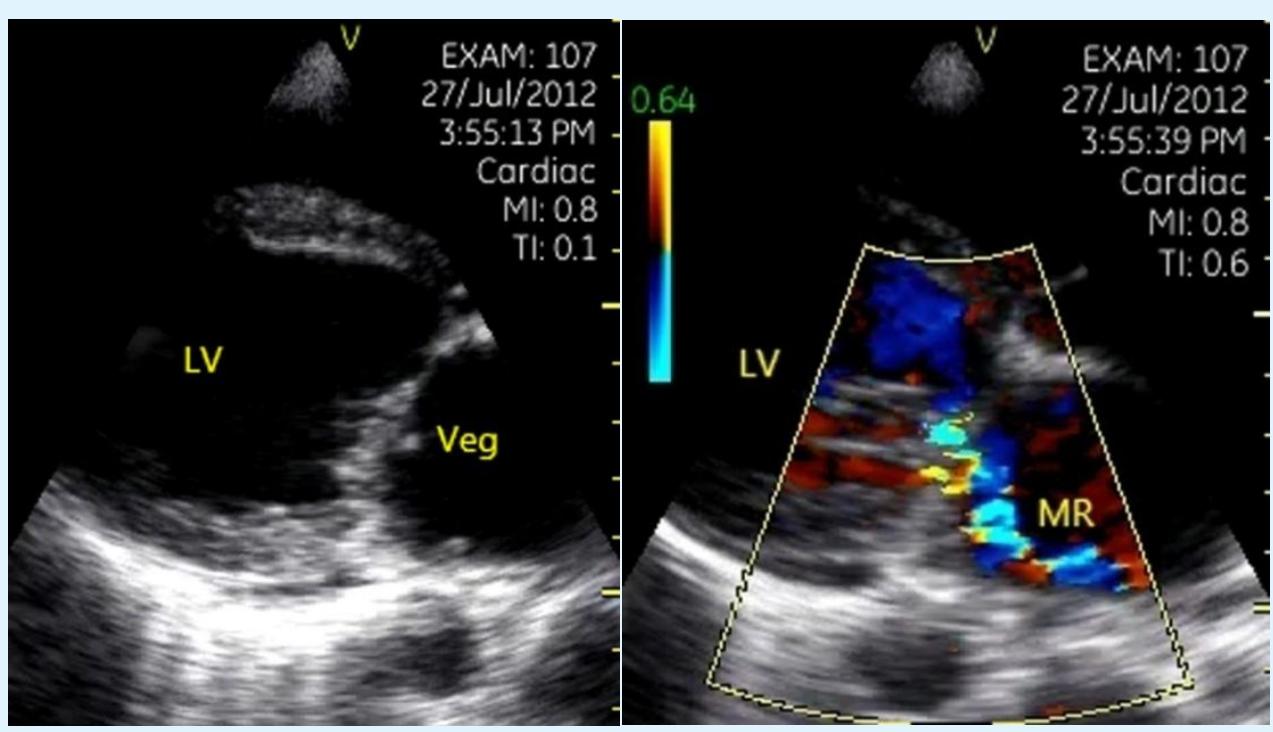

Figure 3: Vegetation in mitral valve. Left: There was a small vegetation on the anterior mitral leaflet in parasternal left ventricular long-axis view. Right: Color flow imaging showed significant mitral regurgitation (MR). LV= left ventricle, Veg= vegetation. 


\section{Open Access Journal of Cardiology}

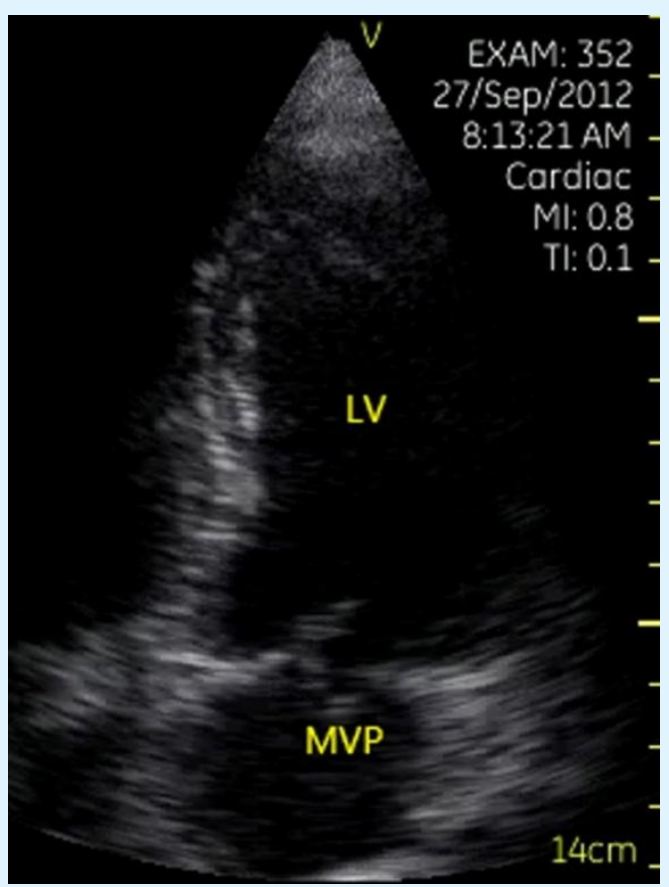

Figure 4: Mitral prolapse. Apical four chamber view showed significant mitral prolapse on posterior leaflet.

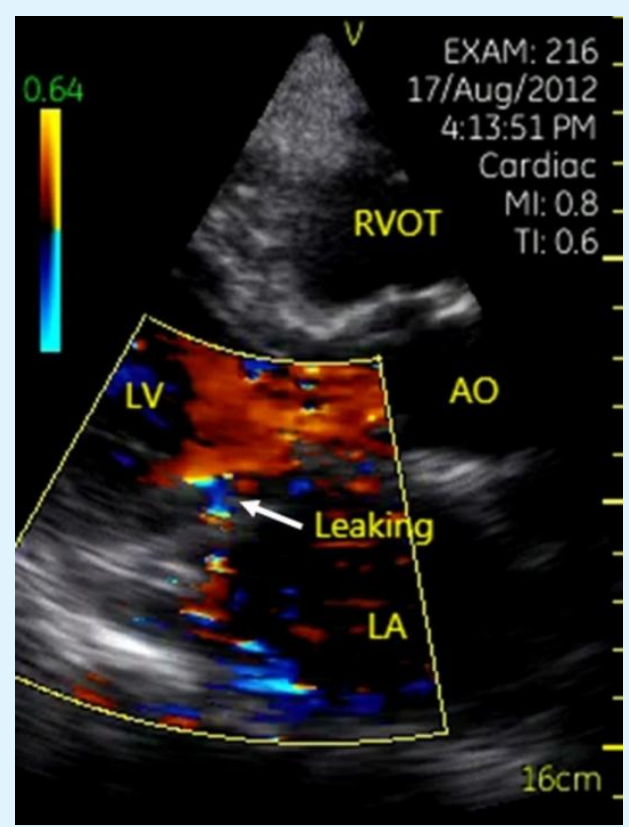

Figure 5: Paravalvular leaking after prosthetic mitral valve replacement. Color flow imaging showed a paravalvular leaking (arrow) in the parasternal left ventricular longaxis view.

\begin{tabular}{|c|c|c|c|c|}
\hline & PE & $\mathbf{C E}$ & $\begin{array}{c}\text { Physical examination } \\
\text { finding* }\end{array}$ & $\begin{array}{c}\text { Agreement between PE vs. CE } \\
\text { (kappa value) }\end{array}$ \\
\hline \multicolumn{5}{|c|}{ Major findings } \\
\hline LV enlargement & 36 & 36 & $\mathrm{~N} / \mathrm{A}$ & $100 \%(1.000)$ \\
\hline LA enlargement & 68 & 71 & N/AW & $96 \%(0.967)$ \\
\hline RV enlargement & 16 & 18 & $\mathrm{~N} / \mathrm{A}$ & $89 \%(0.936)$ \\
\hline RA enlargement & 19 & 19 & $\mathrm{~N} / \mathrm{A}$ & $100 \%(1.000)$ \\
\hline LV dysfunction $(\mathrm{LVEF}<55 \%)$ & 45 & 45 & $\mathrm{~N} / \mathrm{A}$ & $100 \%(1.000)$ \\
\hline $\begin{array}{c}\text { LV hypertrophy (male }>10 \mathrm{~mm} \text {, } \\
\text { female }>9 \mathrm{~mm})\end{array}$ & 41 & 39 & $\mathrm{~N} / \mathrm{A}$ & $97 \%(0.969)$ \\
\hline Valve stenosis (any) & 2 & 2 & 1 & $100 \%(1.000)$ \\
\hline $\begin{array}{l}\text { Valve regurgitation> } \\
\text { mild }\end{array}$ & 98 & 99 & 20 & $99 \%(0.989)$ \\
\hline Mitral valve prolapse & 4 & 4 & 3 & $100 \%(1.000)$ \\
\hline Valvular vegetation & 2 & 2 & $\mathrm{~N} / \mathrm{A}$ & $100 \%(1.000)$ \\
\hline Intracardiac thrombus & 4 & 5 & $\mathrm{~N} / \mathrm{A}$ & $80 \%(0.886)$ \\
\hline Cardiac Mass & 3 & 3 & $\mathrm{~N} / \mathrm{A}$ & $100 \%(1.000)$ \\
\hline Congenital abnormalities & $8^{*}$ & 9 & 2 & $89 \%(0.889)$ \\
\hline $\begin{array}{c}\text { Aneurysm/ } \\
\text { dilation of aorta }\end{array}$ & 20 & 26 & $\mathrm{~N} / \mathrm{A}$ & $88 \%$ (0.853) \\
\hline Pericardial effusion & 29 & 33 & $\mathrm{~N} / \mathrm{A}$ & $88 \%(0.923)$ \\
\hline Hypertrophic cardiomyopathy & 8 & 8 & $\mathrm{~N} / \mathrm{A}$ & $100 \%(1.000)$ \\
\hline Total & 403 & 419 & $\mathrm{~N} / \mathrm{A}$ & $96 \%(0.962)$ \\
\hline \multicolumn{5}{|c|}{ Minor findings } \\
\hline Mild aortic regurgitation & 47 & 47 & $\mathrm{~N} / \mathrm{A}$ & $100 \%(1.000)$ \\
\hline
\end{tabular}




\section{Open Access Journal of Cardiology}

\begin{tabular}{|c|c|c|c|c|}
\hline Mild mitral regurgitation & 90 & 107 & N/A & $84 \%(0.831)$ \\
\hline Mild tricuspid regurgitation & 95 & 121 & N/A & $79 \%(0.735)$ \\
\hline Total & 232 & 275 & N/A & $84 \%(0.854)$ \\
\hline
\end{tabular}

Table 3: Physical examination finding and agreement between PE and CE in patients with major and minor abnormalities. NA: none applicable. *: We refer the heart murmur as the physical exam finding.

\begin{tabular}{|c|c|c|c|c|c|c|c|}
\hline & AUC & SE & $\mathbf{9 5 \%}$ CI & Sensitivity & Specificity & $\begin{array}{c}\text { Positive } \\
\text { predictive value }\end{array}$ & $\begin{array}{c}\text { Negative } \\
\text { predictive value }\end{array}$ \\
\hline LV enlargement & 0.986 & 0.01 & $0.959-0.997$ & 97 & 100 & 100 & 94 \\
\hline LA enlargement & 0.979 & 0.01 & $0.948-0.994$ & 96 & 100 & 100 & 97.7 \\
\hline RV enlargement & 0.944 & 0.04 & $0.903-0.972$ & 88.9 & 100 & 100 & 98.9 \\
\hline RA enlargement & 1 & 0 & $0.982-1.000$ & 100 & 100 & 100 & 100 \\
\hline
\end{tabular}

Table 4: The value of PE to evaluate cardiac chamber dimension assessed by CE.

\begin{tabular}{|c|c|c|c|c|c|}
\hline CE PE & Grade 0 & Grade I & Grade II & Grade III & Total \\
\hline Grade 0 & 97 & 3 & 0 & 0 & $\mathbf{1 0 0}$ \\
\hline Grade I & 3 & 56 & 2 & 0 & $\mathbf{6 1}$ \\
\hline Grade II & 1 & 2 & 26 & 0 & $\mathbf{2 9}$ \\
\hline Grade III & 0 & 0 & 1 & 9 & $\mathbf{1 0}$ \\
\hline Total & $\mathbf{1 0 1}$ & $\mathbf{6 1}$ & $\mathbf{2 9}$ & $\mathbf{9}$ & $\mathbf{2 0 0}$ \\
\hline
\end{tabular}

Table 5: The severity of valve regurgitation evaluated by PE was highly agreed with CE (Kappa= 0.905).

Eight of 9 patients with congenital heart disease could be diagnosed by $\mathrm{PE}$, included 1 patient with left type transposition of great artery (L-TGA) (Figures 6a \& 6b), 3 patients with atrial septal defect (ASD), 2 patients with interventricular septal defect (VSD, (Figure 7)), and 2 patients with ASD or VSD after transcatheter closure procedures (Figure 8). One patient with coronary fistula was failed to diagnose because of bad image quality.

Eight patients with critical ills in emergency room had been correctly diagnosed by PE, included 1 patient with dissection of ascending aorta, 1 patient with a huge pseudo-arterial aneurysm 3 years after aortic Bentall procedure (Figure 9), 1 patient with severe pericardial effusion and tamponade, 1 patient with severe pericardial effusion and chronic exudative pericarditis (Figure 10), 1 patient with acute myocardial infarction and untypical electrocardiogram, 2 patients with rupture of the left ventricular wall after acute myocardial infarction (Figure 11) and 1 patient with left atrial thrombusis confirmed by transesophageal echocardiography and computed tomography angiology (Figures 12a-12c).

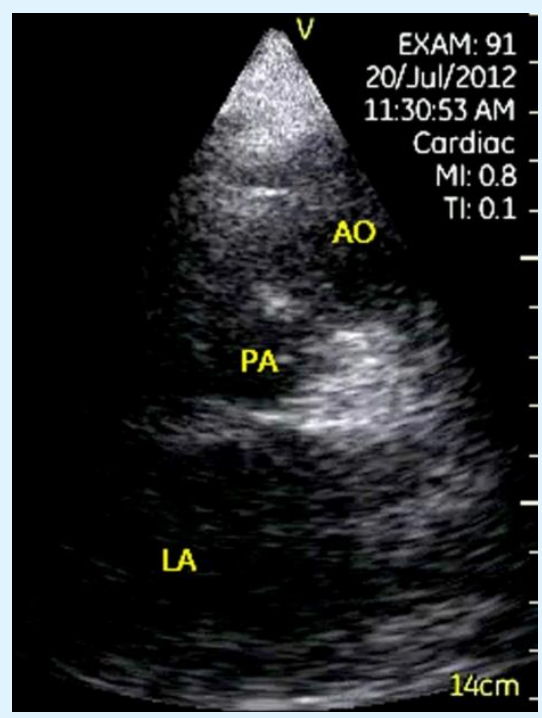

Figure 6: Left- type transposition of great artery performed. Left: Aorta and pulmonary artery paralleled in anterior and posterior direction in apical long-axis view of left ventricle. Right: Parasternal short-axis view of aorta showed that the aorta located in left and anterior of the pulmonary artery. $\mathrm{AO}=$ aorta, $\mathrm{PA}=$ pulmonary artery, $\mathrm{LA}=$ left atrium. 


\section{Open Access Journal of Cardiology}

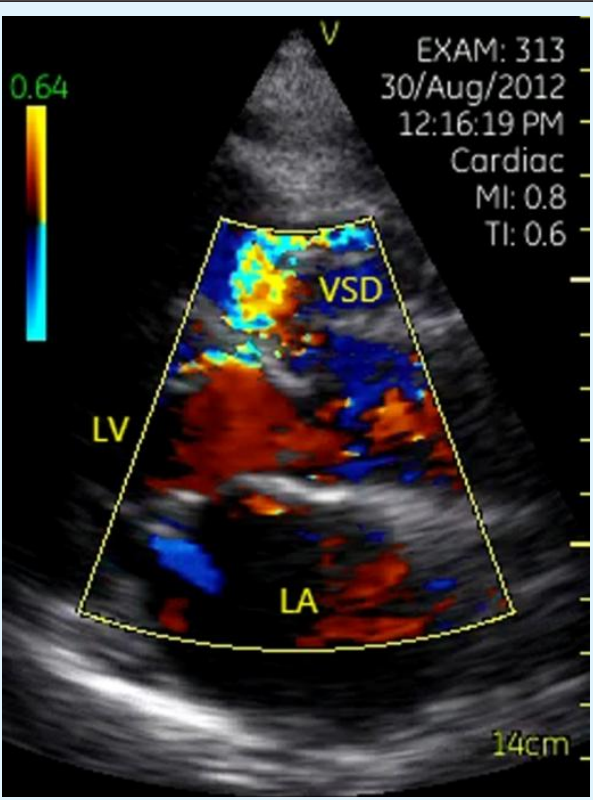

Figure 7: Interventricular septal defect. Parasternal left ventricular long-axis view showed a turbulent flow from left ventricle to right ventricle through the interventricle spetal defect. VSD= interventricular septal defect, $\mathrm{LV}=$ left ventricle, $\mathrm{LA}=$ left atrium.

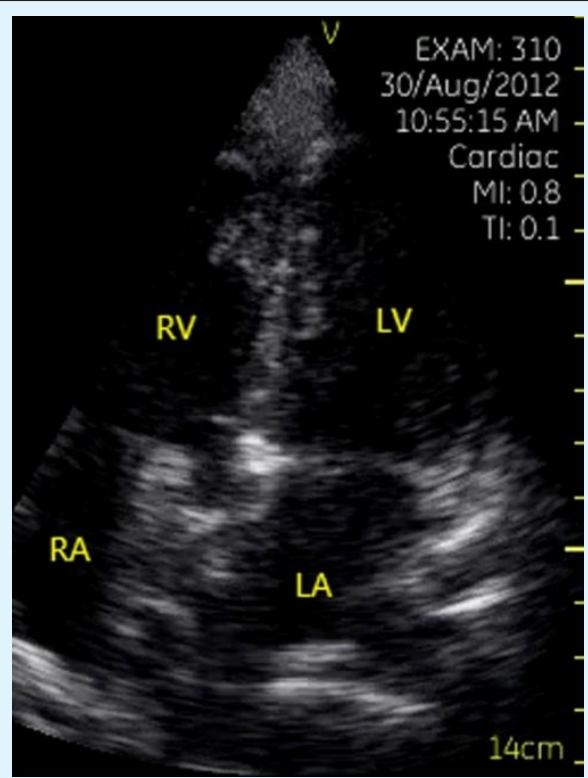

Figure 8: Carpentier device after transcatheter interatrial septal defect closure. Apical four-chamber view showed a device on the interatrial septum between right and left atrium. $\mathrm{LV}=$ left ventricle, $\mathrm{LA}=$ left atrium, $\mathrm{RV}=$ right ventricle, $\mathrm{RA}=$ right atrium.
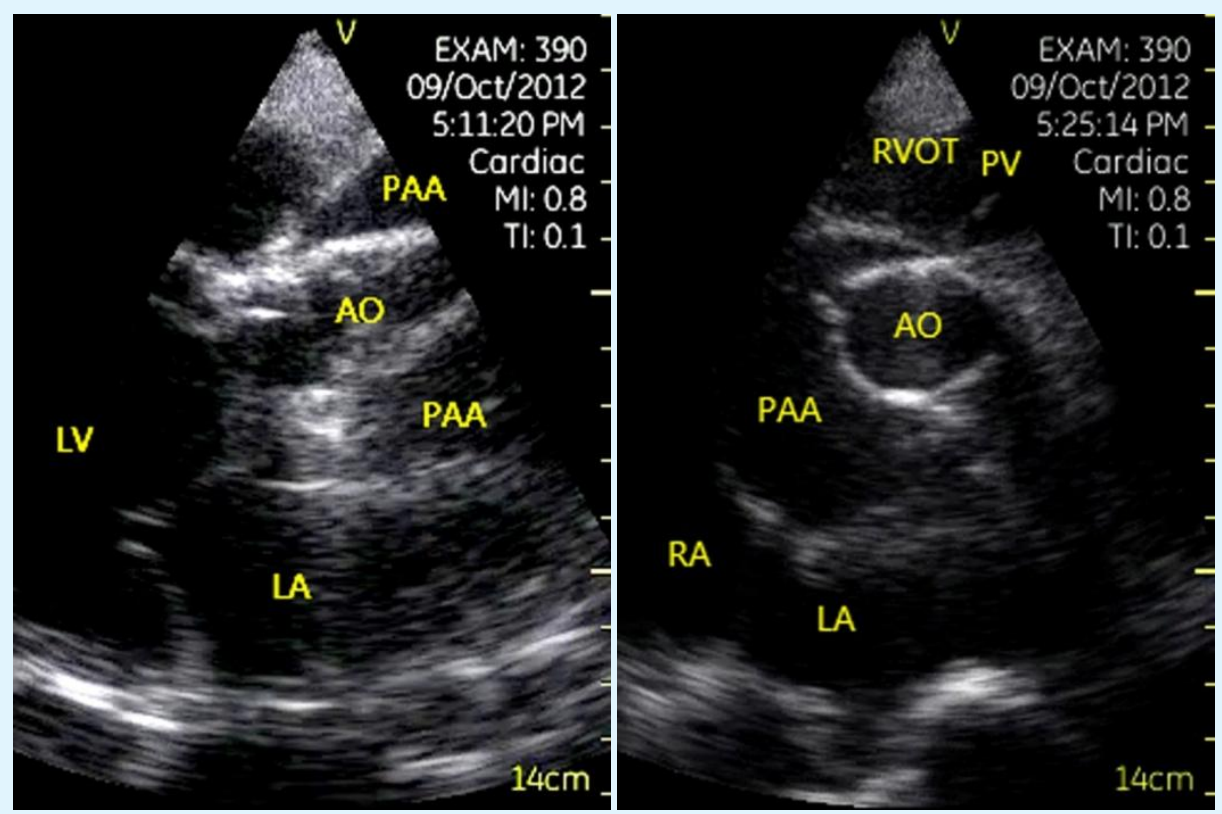

Figure 9: Pseudo arterial aneurysm after Bentall procedure 3 years in patient with Behçet's disease. Left: Parasternal left ventricular long-axis view. Right: Parasternal short-axis view of aorta. PAA= pseudo arterial aneurysm, $L V=$ left ventricle, $\mathrm{LA}=$ left atrium. 


\section{Open Access Journal of Cardiology}

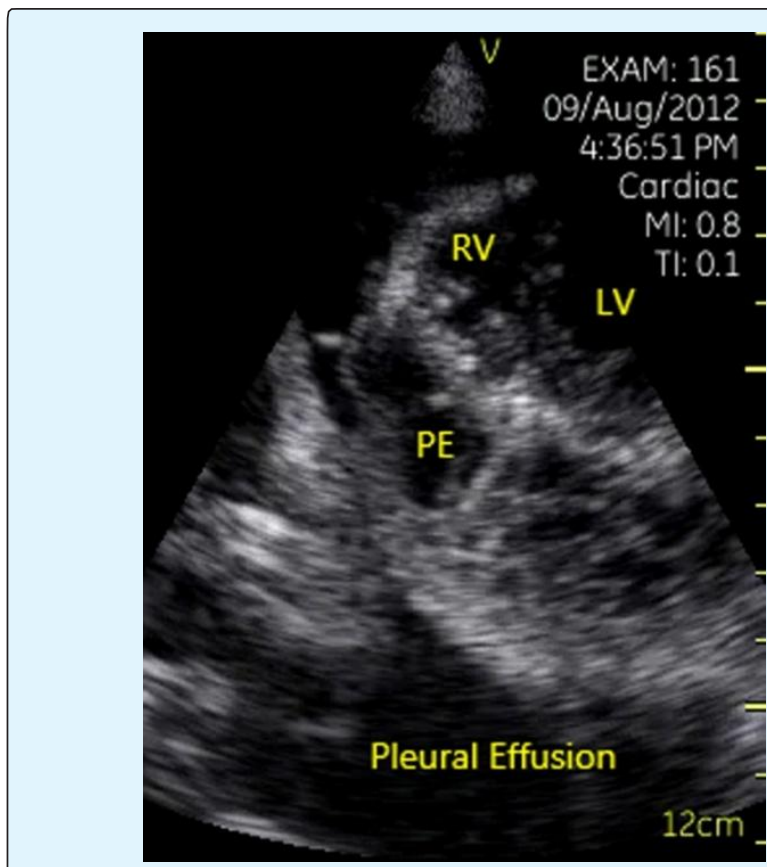

Figure 10: Large amount of pericardial effusion and chronic exudative pericarditis. Parasternal short-axis view of left ventricle at apical level showed a large amount of pericardial effusion and honeycomb-like structure in pericardium. $\mathrm{PE}=$ pericardial effusion, $\mathrm{LV}=$ left ventricle, $\mathrm{RV}=$ right ventricle.

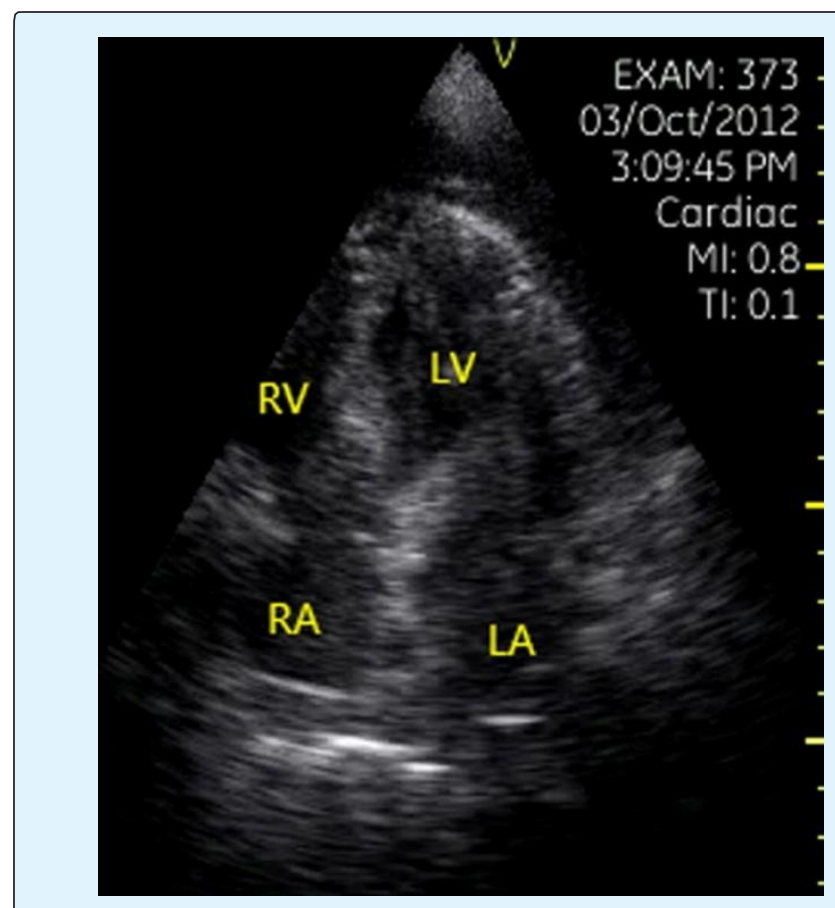

Figure 11: Rupture of left ventricular free wall. Apical four-chamber view showed a large amount of thrombus filled in whole heart chamber.

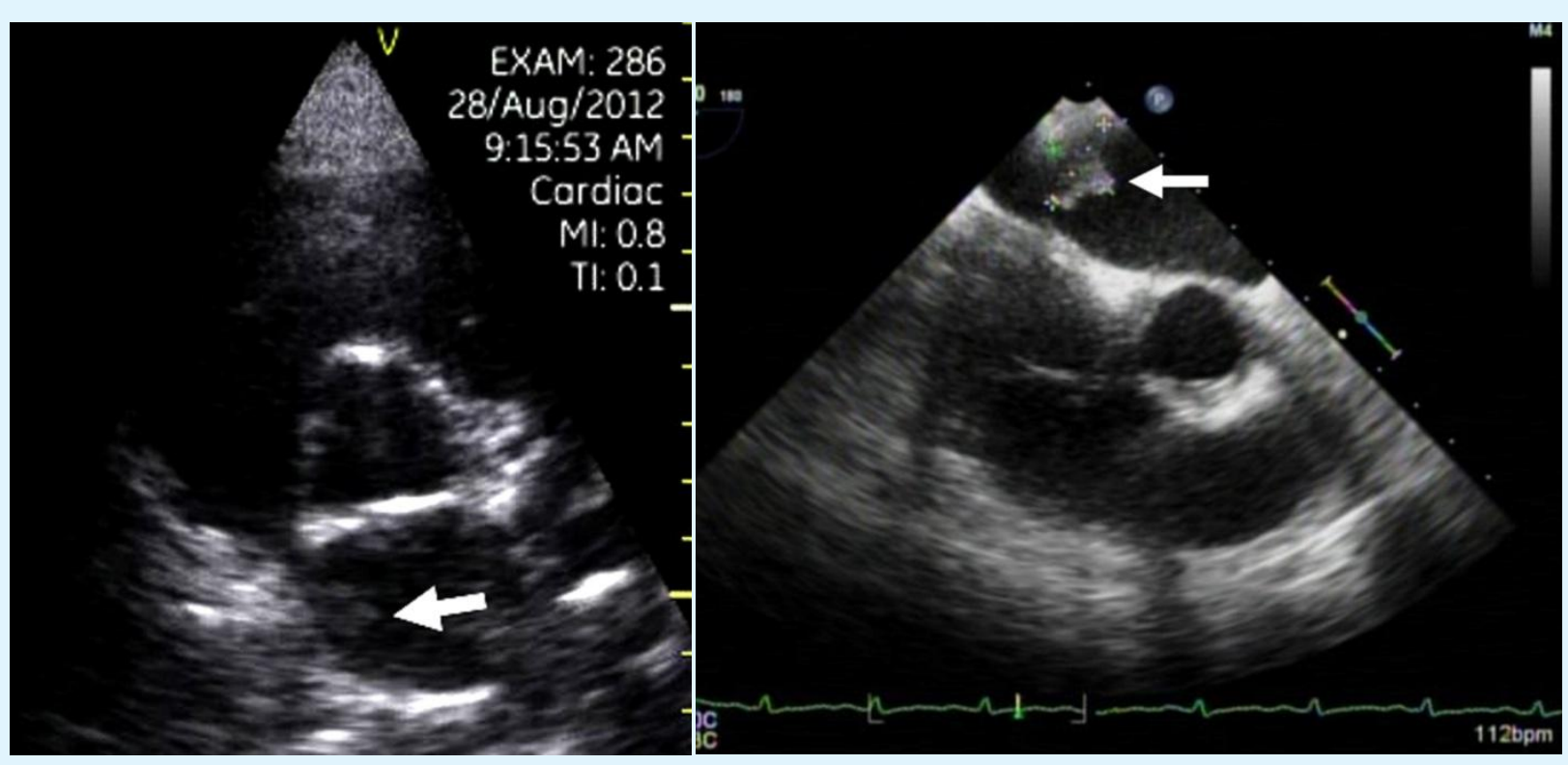

Figure 12: Left atrial thrombus. Left: Parasternal short-axis view of aorta showed a small thrombus on the basal of left atrial wall which closed to the interatrial septum and posterior wall of the left atrium. Middle: Trans esophageal echocardiography showed a thrombus in the left atrium. Right: The thrombus was confirmed by CT (arrow). 


\section{Open Access Journal of Cardiology}

Two hundred thirty-two of 275 (84\%) minor abnormalities were successfully detected by PE (kappa= $0.854)$. Only $17 / 107$ (16\%) mild mitral regurgitation and 26/121 (21\%) mild tricuspid regurgitation missed by PE.

\section{Discussion}

Previous reports showed that PE had greater advantages compared to $\mathrm{CE}$ and hand-carried echocardiographic device [9-13]. It may due to pocket size nature, good enough image quality and true frame of color flow imaging of PE. PE is suitable to study in the emergency and operation room, outside hospital consultation and ward rounds in anytime, especially in patient with critical ills.

In this study, we evaluate the image quality, capability to answer clinical questions, the value of $\mathrm{PE}$ in evaluation of cardiac chamber enlargement, valvular heart disease and congenital heart disease, global and segmental cardiac function. PE could answer over $77.5 \%$ clinical configured questions and provide un configured cardiac findings at the first stop which could not answer by physical examination and may result in changes on clinical decision making and management. The value of PE to evaluate the major abnormalities was similar to the CE, included for evaluating the size of cardiac chamber, valvular heart disease, hypertrophic cardio myopathy and congenital heart disease. PE may become a new tool to modify the one-stop consultation model. We hope that the new one-stop consultation model in the future would be included interrogation, physical examination and PE.

We successfully diagnosed 8 patients with critical ills by PE in emergency room. This first hand information at emergency room had great impacts in evaluation of patient prognosis, clinical decision making and acute management.

PE could be used in follow-up for patients with decreased blood pressure after ablation of atrial fibrillation, Tran's catheter ASD or VSD closure and coronary intervention to find possible complications after procedure. With its advantage of portable size and acceptable image quality, PE could meet the need for outside hospital consultation and provided practical suggestions.

However, $\mathrm{PE}$ is not designed to replace $\mathrm{CE}$. The $75^{\circ}$ sector angle is narrower compared to current CE, and our $\mathrm{PE}$ user is clinical physician but not sonographer, who insufficiency of operator experience render physician overlook the tiny clue of structure abnormalities. The missing detected pericardial effusion and dilation of ascending aorta were mainly due to operator experience. In other reports, the physician experience felled far short [10]. As ASE guideline recommended that it is necessary for physician to undergo level II training in echo laboratory [5].

Limitation: We enrolled a large percentage of patients with left ventricular diastolic dysfunction or pulmonary hypertension because of consecutive patient nature. Therefore, clinical questions answered by PE were significantly less than CE. Finally, the suspected findings and unanswered clinical questions by PE still need CE and Transoesophageal echocardiography examination. Moreover, the detection of diastolic function, flow velocity, pulmonary systolic pressure is not available with PE range.

\section{Conclusions}

PE allows immediately detecting the intra cardiac structure and flow, had almost perfect performance in finding major and minor abnormalities associated with great concordance with CE. The pocket size echocardiographic device would like a stethoscope for cardiologist during consultation and determine release from the outpatient clinic which helps them to diagnose and manage critically ill patients at the bedside. Being ultra-portable and convenient, PE may become part of the clinical examination, performing like an excellent consultation tool.

With pocket size nature and good agreement with CE, PE showed additive clinical value over the clinical physical examination. It may alter the current consultation mode in clinical practice in the future.

\section{Disclosures}

This article was supported by GE Healthcare, the Natural Science Foundation of China [Grant number 30800453 and 81270206], Shanghai Rising-Star Program [Grant number 10QA1404500], Natural Science Foundation of Shanghai [Grant number 12ZR1417600] and Shanghai Med-X program [Grant number YG2010MS29].

The author gratefully acknowledges the support of K. C. Wong Education Foundation. 


\section{Open Access Journal of Cardiology}

\section{References}

1. Giannotti G, Mondillo S, Galderisi M, Barbati R, Zaca V, et al. (2005) Hand-held echocardiography: Added value in clinical cardiological assessment. Cardiovasc Ultrasound 3: 7.

2. Mondillo S, Giannotti G, Innelli P, Ballo PC, Galderisi M (2006) Hand-held echocardiography: Its use and usefulness. Int J Cardiol 111(1): 1-5.

3. Spencer KT, Anderson AS, Bhargava A, Bales AC, Sorrentino M, et al. (2001) Physician-performed point-of-care echocardiography using a laptop platform compared with physical examination in the cardiovascular patient. J Am Coll Cardiol 37(8): 20132018.

4. Salustri A, Trambaiolo P (2002) Point-of-care echocardiography: Small, smart and quick. Eur Heart J 23(19): 1484-1487.

5. Seward JB, Douglas PS, Erbel R, Kerber RE, Kronzon I, et al. (2002) Hand-carried cardiac ultrasound (hcu) device: Recommendations regarding new technology. A report from the echocardiography task force on new technology of the nomenclature and standards committee of the american society of echocardiography. J Am Soc Echocardiogr 15(4): 369373.

6. Quinones MA, Otto CM, Stoddard M, Waggoner A, Zoghbi WA, et al. (2002) Recommendations for quantification of doppler echocardiography: A report from the doppler quantification task force of the nomenclature and standards committee of the american society of echocardiography. J Am Soc Echocardiogr 15(2): 167-184.
7. Zoghbi WA, Enriquez-Sarano M, Foster E, Grayburn PA, Kraft CD, et al. (2003) Recommendations for evaluation of the severity of native valvular regurgitation with two-dimensional and doppler echocardiography. J Am Soc Echocardiogr 16(7): 777802 .

8. Landis JR, Koch GG (1977) An application of hierarchical kappa-type statistics in the assessment of majority agreement among multiple observers. Biometrics 33(2): 363-374.

9. Prinz C, Voigt JU (2011) Diagnostic accuracy of a hand-held ultrasound scanner in routine patients referred for echocardiography. J Am Soc Echocardiogr 24(2): 111-116.

10. Choi BG, Mukherjee M, Dala P, Young HA, Tracy CM, et al. (2011) Interpretation of remotely downloaded pocket-size cardiac ultrasound images on a webenabled smartphone: Validation against workstation evaluation. J Am Soc Echocardiogr 24(12): 13251330.

11. Cardim N, Fernandez Golfin C, Ferreira D, Aubele A, Toste J, et al. (2011) Usefulness of a new miniaturized echocardiographic system in outpatient cardiology consultations as an extension of physical examination. J Am Soc Echocardiogr 24(2): 117-124.

12. Goodkin GM, Spevack DM, Tunick PA, Kronzon I (2001) How useful is hand-carried bedside echocardiography in critically ill patients? J Am Coll Cardiol 37(8): 2019-2022.

13. Vourvouri EC, Poldermans D, Deckers JW, Parharidis GE, Roelandt JR (2005) Evaluation of a hand carried cardiac ultrasound device in an outpatient cardiology clinic. Heart 91(2): 171-176. 